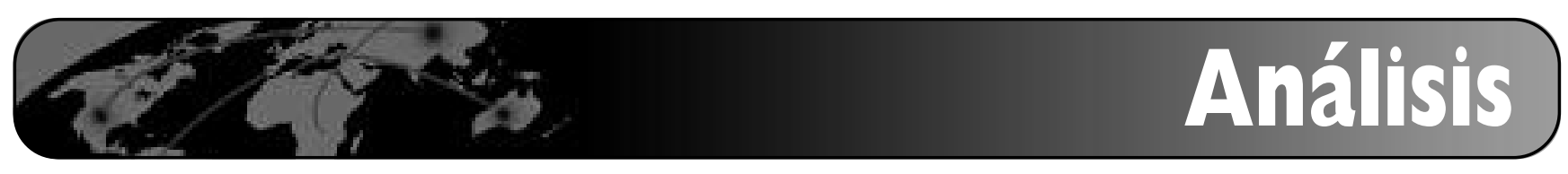

\title{
Ferroatlántica I+D y la vigilancia tecnológica
}

\author{
Por Lara Rey Vázquez
}

Resumen: En este artículo se explica como Ferroatlántica $I+D$ ha implantado con éxito un sistema de vigilancia tecnológica e inteligencia competitiva. Ferroatlántica se dedica a la fabricación de ferroaleaciones, y Ferroatlántica $I+D$ tiene como objetivo fundamental el desarrollo y la coordinación de la actividad investigadora del grupo. El sistema tiene por objetivo disponer, de manera continuada, de la información necesaria sobre cualquier tema que en ese momento se esté tratando, contribuyendo con información que dé soporte y fundamente la resolución de temas propios de $I+D$.

Palabras clave: Ferroatlántica, Vigilancia tecnológica, Inteligencia para la $I+D+I$, Gestión de la información

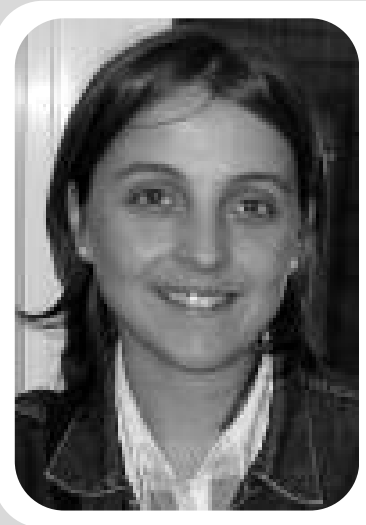
Licenciada en geografía e historia con especialidad en biblioteconomía y documentación (1997) por la Universidad de Santiago de Compostela. Actualmente es la encargada del Departamento de Documentación y Proyectos de la empresa Ferroatlántica $I+D$. Tiene formación postgradual en materias relacionadas con la gestión de información, tecnologías de la información y comunicación, vigilancia tecnológica y propiedad intelectual. http://www.interneteando-lara. blogspot.com tecnológica.

\section{Title: Ferroatlántica $R+D$ \& the technology watch}

Abstract: The article presents a case study of Ferroatlántica $R \& D$, showing how a technology watch and competitive intelligence system has been successfully implemented. Ferroatlántica is a ferroalloy manufacturer, and Ferroatlántica $R \& D$ 's main purpose is to develop and coordinate the group's research activities. The most important objective of the system is to make available on a continual basis the information needed on any topics currently being addressed. The information provided supports and provides a base for resolving specific $R \& D$ issues.

Keywords: Ferroatlántica, Technology watch, Technical intelligence, Information management, Intelligence process.

Rey Vázquez, Lara. "Ferroatlántica I+D y la vigilancia tecnológica". En: El profesional de la información, 2006, noviembre-diciembre, v. 15, n. 6 , pp. $420-425$.

FERROATLÁNTICA SL, ES UNA EMPRESA CREADA EN 1992 de capital $100 \%$ privado y español, perteneciente al Grupo Espacio. Tiene fábricas en Galicia (A Coruña, Cee y Dumbría), Cantabria (Boo de Guarnizo), Aragón (Monzón) y Venezuela (Puerto Ordaz) y cuya dirección se centraliza en Madrid. La empresa cuenta con unas 600 personas en plantilla.

Es la única empresa española que se dedica a la fabricación de ferroaleaciones y la segunda en la Unión Europea. Las ferroaleaciones son aditivos que se agregan al acero para darle unas mejores propiedades físicas, y el silicio metal se usa para alear con el aluminio y para hacer siliconas. Se trata de una industria básica, es decir, partiendo de unas materias primas se fabrica un producto semielaborado que se comercializa como aditivo o aleación de otros productos.

En este grupo se integra Ferroatlántica $I+D$ que tiene como objetivo fundamental desarrollar y coordinar la actividad investigadora del grupo, aunando esfuerzos en una línea común. Nació como resultado de un proyecto de investigación que se llevó a cabo en la fábrica de Sabón, en Arteixo (A Coruña), en el año 1995 y que culminó con una patente que modificó el proceso de producción del silicio metal. La demanda de esta tecnología a nivel mundial desembocó en la creación de Ferroatlántica $I+D$. Su personal ha mejorado e implantado a través de royalties la patente del Electrodo ELSA ${ }^{1}$ por todo el mundo. A raíz de este proyecto, se han realizado otros muchos que han llevado a Ferroatlántica a ser el primer productor del mundo (datos del año 2005).

\section{¿Qué es la VT?}

Un sistema de vigilancia tecnológica (VT) puede definirse como la búsqueda, detección, análisis y comunicación a los directivos de la empresa de informaciones orientadas a la toma de decisiones sobre amenazas y oportunidades externas en el ámbito de la ciencia y la tecnología (Escorsa). Según Morcillo 
consiste en analizar el comportamiento innovador de los competidores directos e indirectos, explorar todas las fuentes de información (libros, bases de datos, patentes, etc.), examinar los productos existentes en el mercado (tecnología incorporada) y asistir a ferias y congresos para posicionarse respecto a los demás competidores y tomar así conocimiento de las competencias tecnológicas que predominarán en un futuro más o menos próximo. Todo ello sin perder de vista la capacidad tecnológica presente y la que estará en condiciones de desarrollar la empresa para enfrentarse a nuevos retos.

La vigilancia debe ser:

-Focalizada: selección de áreas, tecnologías e indicadores.

- Sistematizada: seguimiento y exploración de los indicadores.

-Estructurada: organización interna y evolución continua de sus diferentes fases (observación, captación, análisis, difusión, seguimiento y control).

Con esto el sistema contribuirá definir la posición estratégica que ha de tomar la empresa y sus objetivos en $\mathrm{I}+\mathrm{D}$, permitiendo:

- Realizar de manera sistemática la observación y búsqueda de señales de cambio y novedades enfocadas a la captura de información, la selección y análisis, la difusión y comunicación para convertirla en conocimiento.

- Alertar sobre innovaciones.

- Investigar los hallazgos realizados para la ejecución de nuevos procesos.

- Buscar soluciones tecnológicas.

\section{Ferroatlántica I+D y la VT}

El mundo de las ferroaleaciones es un sector donde todos se conocen y donde cada uno sabe aproximadamente lo que está haciendo su competidor. Los competidores de
Ferroatlántica en el mundo, grandes multinacionales, no vieron venir el resultado de la investigación que se estaba llevando a cabo hasta que se patentó. Muchas empresas tuvieron que comprar la tecnología y pagar los correspondientes royalties por no haber detectado a tiempo las señales débiles que había en el entorno.

La vigilancia está estrechamente unida a la gestión de la innovación y a la estrategia de la empresa. Sin la existencia de una previa reflexión estratégica difícilmente cabe plantearse un esfuerzo de articulación de la vigilancia. Se proyecta sobre la toma de decisiones empresariales alertando sobre posibles amenazas y oportunidades, aportando nuevos elementos y enfoques, y reduciendo el riesgo a la hora de la toma de decisiones.

Los diferentes proyectos que iban surgiendo, la necesidad de vigilar en la medida de lo posible a los competidores e intentar agluti-

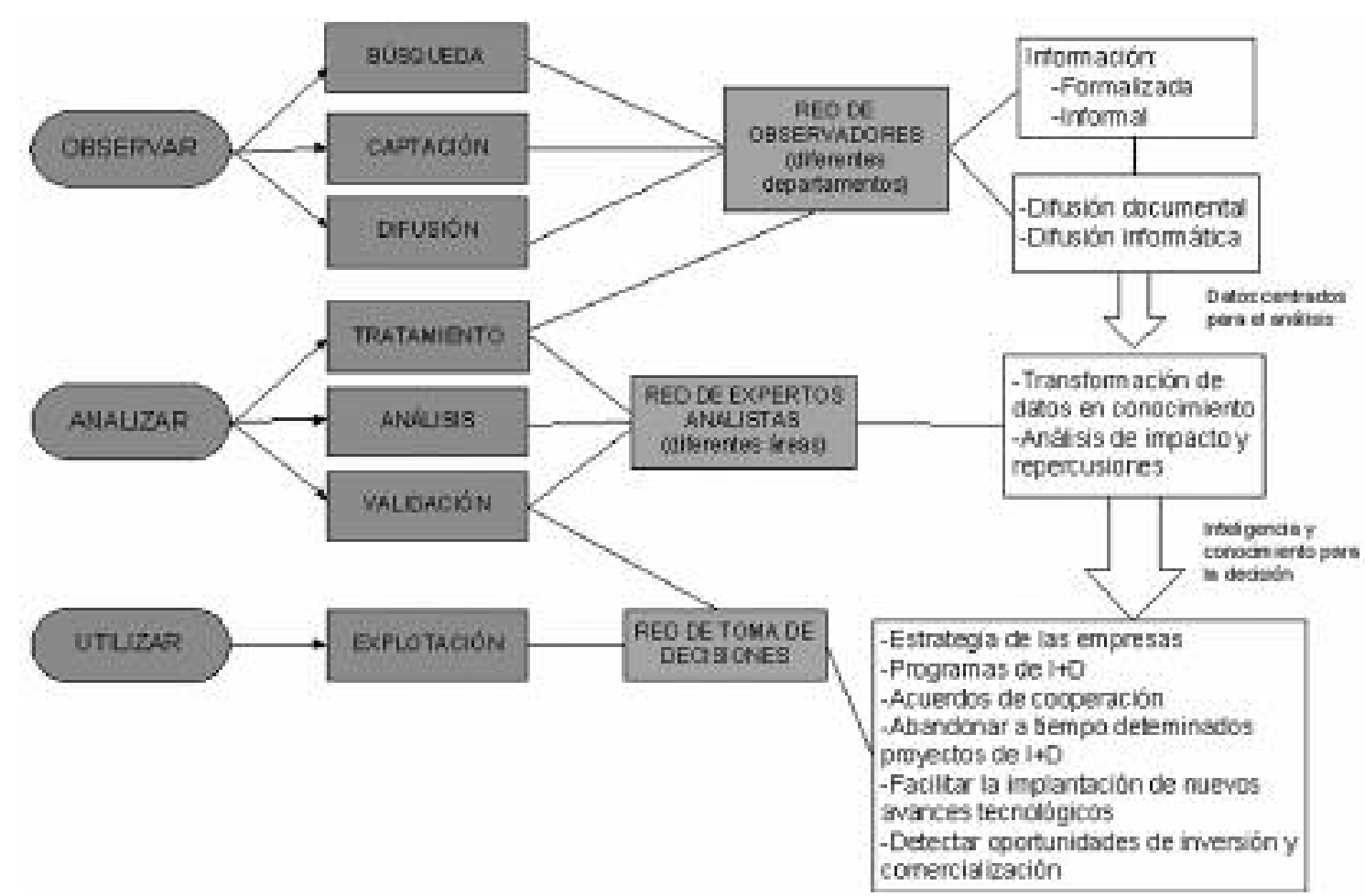

Esquema de: "Desarrollo y puesta en práctica de un sistema de vigilancia tecnológica sectorial para las empresas transformadoras de productos del mar", Anfaco-Cecopesca 
nar todo el conocimiento disponible en una unidad centralizada fue la semilla de la Unidad de Vigilancia Tecnológica de Ferroatlántica $I+D$.

La identificación de las fuentes de información se realizó tomando como base las necesidades de la misma:

-Documentación propia o relacionada con la organización.

- Personas con conocimientos relacionados con la organización.

-Contactos externos de interés.

-Centros de investigación, universidades, etc.

-Fuentes documentales.

-Documentación técnica.

-Congresos, ferias, seminarios.

Basándonos en la identificación de necesidades y en el procedimiento de acceso a las fuentes identificadas, la Unidad de VT incluye un proceso de búsqueda y tratamiento de la información, con un sistema de gestión que permite disponer de ella al momento. Está compuesta por una biblioteca física y por un sistema montado con tecnología web, que se puede consultar desde todos los puntos de la empresa. Se alimenta de tres tipos de información:

\section{Interna}

Es la más valiosa y se ha integrado en el sistema como una parte más del mismo. Organizada en grades temas y con diferentes permisos de acceso es el corazón de todo el modelo. Informes, presentaciones, artículos propios, patentes, normativa interna, etc., se han ido introduciendo poco a poco, y en paralelo se ha organizado un fondo bibliográfico compuesto por monografías, revistas especializadas, proceedings, catálogos que han sido proporcionados por las diferentes fábricas, conformando así una biblioteca organizada. Tiene un for- mulario en línea para realizar búsquedas, con la posibilidad de pedir en préstamo la publicación y también la disponibilidad en texto completo de la gran mayoría de los artículos (más de 1.500).

Los boletines sectoriales a los que estaba suscrita la empresa se han ido cambiado, en la medida de lo posible, a suscripción electrónica para que de esta manera su distribución sea más sencilla y así lleguen a todos los interesados sin necesidad de realizar tediosas copias en papel, como se hacía anteriormente.

\section{Patentes (seguimiento pro- pio)}

Han sido siempre una fuente de información cotizada, compleja y no siempre localizable (los títulos de las patentes muchas veces de manera intencionada, no reflejan el contenido real y además muchos países no tienen de una manera clara el acceso a sus bases de datos).

Tener la posibilidad de usarlas ayuda a conocer el estado de la técnica, favoreciendo no duplicar investigaciones (con el gasto que puedan suponer), resuelven problemas concretos, detectan nuevas tecnologías que aún no han sido publicadas, y facilita localizar socios. Todo esto unido a la vigilancia de la competencia, permite realizar un análisis de mercado y de tendencias muy completo.

\section{"La vigilancia es un soporte indispensable para la toma de decisiones a medio y largo plazo" (Robert J. Mockler)}

Como fuente de información principal en la VT, las familias de
El profesional de la información está abierto a todos los bibliotecarios, documentalistas y otros profesionales de la información, así como a las empresas y organizaciones del sector para que puedan exponer sus noticias, productos, servicios, experiencias y opiniones.

Dirigir todas las colaboraciones para publicar a:

El profesional de la información

Apartado 32.280

08080 Barcelona epi@elprofesionalde lainformacion.com

patentes $^{2}$ y su clasificación internacional, así como las labores de difusión que se están haciendo, han acercado a las empresas una documentación muy valiosa. Hay que pensar que la patente es, por definición, la primera publicación de una invención (no ha sido publicada antes en ningún otro medio, pues de ser así automáticamente ya no sería patentable) e identifica de una manera clara al inventor y al titular.

De todas maneras hay que tener en cuenta que su valor como fuente de información depende del grado de empleo y su importancia para la estrategia del sector de que se trate. Pensemos que muchas multinacionales patentan para desviar la atención sobre la tecnología que realmente están investigando. De esta forma la competencia se desorienta y pierde el tiempo tratando de descifrar esos señuelos.

La búsqueda, selección y organización de patentes es quizá una de las tareas que más tiempo lleva y 
en la que se involucra a más gente. En el equipo de Ferroatlántica $I+D$ esta operación se encuentra coordinada por el director de I+D para que no se dupliquen trabajos. Es una parte tan fundamental, que la asignación de recursos es cada vez mayor.

Un vez localizada una patente y se lee, se trata de identificar lo más significativo haciendo un resumen y se enlaza con otras que ya estén en la base de datos, de manera que las opiniones sobre ella y con otras que tengan una temática similar o un proceso parecido estén relacionadas entre sí. Esto genera una gran riqueza de conocimiento.

\section{Internet (todo un mundo)}

Para las búsquedas puntuales de información, así como para aquellas que requieren una mayor elaboración, se utilizan fuentes disponibles en la Red. En la mayoría de los casos se intenta usar recursos gratuitos, aunque no se descartan los de pago.

Utilidades como CDTracker, Alertas de Google News, TracerLock, AvisaBOE, Copernic, Spypress, HuginOnline, Karnak, y alertas vía correo electrónico (Enervia, Energuia, Ayudas.net, EnergiasRenovables, Titulares, Redaccion Aimme, Madri+d, Centro de Transferencia de Tecnología Galactea, etc.) boletines gratuitos (Unesa, $C N E$, etc.) facilitan enormemente esta tarea.

Podemos identificar tres grupos en función de su valor dentro de la empresa:

a. Difusa. Por supuesto la más difícil de detectar, y al mismo tiempo la que tiene más valor. Puede conseguir modificar el desarrollo de un proyecto. Muchas veces llega a nosotros de forma indirecta $y$ cuesta identificarla ya que una vez detectada no es fácil cotejarla. Normalmente se encuentra dentro de documentación difícil de localizar: patentes, tesis, artículos científicos, actas de congresos especializados, papers, etc.

b. Latente. Se encuentra disgregada y no es de difícil localización, pero sí es complicado unificarla. Suele aparecer en fuentes diferentes $\mathrm{y}$ con formatos diversos. Se puede utilizar para documentar el estado de la técnica, sin hacer un análisis profundo de los resultados obtenidos. Para localizar información de este tipo podemos recurrir a los clientes, a bases de datos de proyectos de I+D, a buscadores especializados, foros, etc.

c. Del entorno. De carácter generalista, de poco valor para la estrategia de la empresa, pero que marca una pauta, o ayuda a ver la tendencia del entorno. Está formada por noticias de prensa, novedades de las ferias, catálogos de proveedores, etc.

\section{Proceso de búsqueda y análisis \\ de la información en internet}

Como afirma Oppenheim (2000), el problema fundamental de la www es su gran volumen, junto a la heterogeneidad e inconsistencia de la información.

Partiendo de la base de que tenemos ya una unidad de VT organizada $^{3}$, vamos a describir cómo sería un proceso de búsqueda y análisis de la información. Para ello utilizaremos como punto de partida internet, aunque finalmente y en virtud de los resultados obtenidos, se puede complementar con la información interna disponible (en nuestro caso aparte de la biblioteca digital, tenemos indexados con Adobe $\mathrm{Ca}$ talog todos los boletines especializados en nuestro sector, que vamos recibiendo en pdf desde el año 1999). El almacenamiento de la información externa sobre el entorno crea una base de datos actualizada e histórica que puede ayudar en gran medida a la toma de decisiones.

\section{Búsqueda de información convencional vs internet}

Al plantearnos una búsqueda en un entorno estructurado, como puede ser cualquier base de datos, el planteamiento tiene una coherencia espacio-temporal. Conocemos a priori su contenido, su acotación cronológica y la delimitación de materiales. Pero con internet este planteamiento no sirve.

Los contenidos tienen otras características:

—Vida limitada de los enlaces (esto ocurre cada vez menos, pero siempre alguno acaba fallando).

-Dinamismo de los contenidos (actualizaciones incluso diarias).

-Democratización de los autores (la Red es accesible a todo el que quiera publicar algo).

- "Nuevas" formas de difusión de información: los blogs especializados, listas de distribución, etc.

-Multitud de resultados sólo válidos en apariencia.

- La denominada "internet invisible".

\section{Estrategia}

La base cultural que requieren la VT y la inteligencia competitiva es la de captar, asimilar y compartir información, esto es, cultura informacional (Cornella, 1997). Ahora bien, no toda es válida. Es necesario que la que circule tenga significado para sus destinatarios, especialmente los que toman decisiones. La característica esencial para que sea útil y, por tanto, cumpla los fines de la vigilancia, es su valoración.

\section{Pasos a seguir:}

-Búsqueda genérica en un buscador tipo Google.

—Directorios temáticos. 
—Bases de datos.

- Sentido común y un poco de suerte (léase buena intuición).

—Validación de la información por un experto.

Particularmente me gusta utilizar la búsqueda genérica como el comienzo del proceso. Poco profesional y poco estructurada y sistemática pero es, bajo mi punto de vista, la forma de enfrentarse al problema. A priori no se sabe si lo que se está buscando es de dominio público y va a aparecer en medios no especializados. Puede concluir según la iniciemos (esto aunque suene sorprendente, a veces ocurre), o puede orientarnos para ir recopilando palabras clave específicas, portales especializados o artículos técnicos.

Al final una buena búsqueda de información depende un poco de la suerte, de la intuición y de la ayuda del experto en la materia. Sin contar con que hay que tener una metodología establecida si no queremos perdernos en una maraña de datos que no sabemos de dónde han salido.

Como resultado de estas acciones se obtendrán informaciones "formales" que podrán ser complementadas con otras de carácter "informal" tales como, comentarios de un cliente, de un proveedor, respuestas en una entrevista, etc. ${ }^{4}$.

Algunos ejemplos de información que encontramos en internet son:

-Movimientos de importación y exportación de productos.

—Estudios de mercado de sectores e incluso productos.

—Patentes de una tecnología.

-Información sobre políticas de $\mathrm{I}+\mathrm{D}$.

\section{Web invisible y sindicación de contenidos}

\section{Web invisible}

"Hay estimaciones que calculan en 500 veces más grande que el total de la información indizada por los buscadores, la información que permanece invisible en la world wide web, lo que se ha denominado el inmenso océano de la internet profunda"s.

Cada vez hay más buscadores que indizan documentos que antes quedaban perdidos por la Red. Era muchas veces la suerte la que nos hacía llegar a enlaces viudos con algún documento interesante. Pero lo que los buscadores siguen sin indicar, y el software específico para ello cada vez tiene más dificultad, son las páginas que tienen un formulario de búsqueda. Hasta hace muy poco eran completadas a nuestro antojo de forma automática por algún software del mercado, pero poco a poco van evolucionando y necesitan de una persona que cubra manualmente la búsqueda. La introducción de Captchas $^{6}$ obliga cada vez de forma más sofisticada a que sea una persona y no una máquina quien realice la consulta de forma manual.

\section{"Probablemente la vigilancia se convertirá en una actividad tan importante para la empresa como lo son hoy el marketing o la I+D" (Michel Cartier)}

También los blogs especializados son muy buena fuente de información, y aunque hay buscadores que los localizan en tiempo real, por ahora yo los consideraría web invisible.

http://www.technorati.com

\section{Sindicación de contenidos}

Un inciso terminológico: el término sindicación es un barbarismo que proviene del inglés "syndication", habitual en la terminología anglosajona de los medios de comunicación (es lo que hacían desde siempre las agencias de noticias). Como tal, se desaconseja su uso en español, aunque se incorporó rápidamente a la jerga técnica con la popularización de la redifusión de contenidos web. "Sindicación de contenidos", en términos lingüísticos más recomendables, sería pues "redifusión de contenidos".

Se basa en poner parte del contenido de una página web a disposición de otros sitios o suscriptores individuales. El sistema más conocido es RSS, seguido por Atom. Los programas informáticos compatibles con ellos consultan periódicamente una página con titulares que enlazan con los artículos completos en el sitio web original. A diferencia de otros medios de comunicación, los derechos de redifusión suelen ser gratuitos y no suele mediar un contrato entre las partes sino una licencia de aceptación de las normas de uso.

La sindicación podría solucionar el problema de la internet invisible, el gran volumen de información que queda fuera de los motores de búsqueda habituales, que queda inaccesible al usuario medio? .

\section{Conclusión}

Cada vez es más fácil acceder a la información que queremos, con mejores herramientas, mejores aplicaciones y más experiencia. Pero al mismo tiempo hay más información disponible. Y para filtrarla de una forma óptima existen en las empresas las unidades de VT.

El ejemplo de Ferroatlántica $I+D$ no deja de ser uno más en España, pero quizá cuando empezamos con este tema no había tantas organizaciones que tuvieran un de- 
partamento de estas características. Con el paso del tiempo, lo que en los años 90 sonaba raro está cada vez más implantado en el quehacer diario.

\section{Notas}

1. Patente $n^{\circ}$ ES2046098 "Process for continuous manufacture of impurity and iron-free electrodes for electric arc furnaces".

2. Como es sabido, una familia está constituida por las diferentes versiones de una misma patente en distintos países.

3. Rey Vázquez, Lara. "Ferroatlántica I+D: experiencia de una unidad de vigilancia tecnológica". En: Puzzle, revista hispana de la inteligencia competitiva, 2004, mayo-junio, año 3, n. 11

4. Norma española experimental UNE 166006 EX, mayo 2006: Gestión de la I+D+I: sistema de vigilancia tecnológica.

5. http://www.hipertexto.info/documentos/ web_invisib.htm

6. En la Red se libra una batalla entre humanos y máquinas. Los spambots atacan en masa sitios web. ¿Son los Captchas una solución para frenarlos?

Una de las soluciones diseñadas para limitar los ataques de robots son los Captchas (Completely automated public Turing tests to tell computers and humans apart), traducido sería "test de turing para diferenciar máquinas y humanos". El concepto fue desarrollado en la Universidad Carnegie Mellon y denominado así por IBM en el año 2000, a partir del investigador Alan Turing. Según la Wikipedia, se trata de hacer que el humano pruebe su condición.

http://interneteando-lara.blogspot.com/2006_04 _30_interneteando-lara_archive.html http://www.captcha.net/

7. Franganillo, Jorge; Catalán, Marcos Antonio. Bitácoras y sindicación de contenidos: dos herramientas para difundir información. En: BiD, 2005, n. 15.

\section{Bibliografía}

Ayuso Sánchez, M. Josefa. Vigilancia tecnológica y sistemas nacionales de innovación: análisis cuantitativo y de calidad de patentes españolas, 1989-1999. Tesis doctoral. Getafe: Universidad Carlos III de Madrid, Departamento de Biblioteconomía y Documentación, 2001.

Callon, Michel; Courtial, Jean-Pierre; Penan, Hervé. Cienciometría :el estudio cuantitativo de la actividad científica, de la bibliometría a la vigilancia tecnológica. Gijón: Trea, D.L. 1995.

Cartier, Michel. La nouvelle société du savoir et son économie, 1999, 2005.

Cornella, Alfons. La cultura de la información como institución previa a la socidad de la información. Barcelona: Esade, 1997.

http://mailweb.udlap.mx/ yulia/bc110/material/ economia.pdf

Dopico Parada, Ana Isabel; Currás del Valle, Consuelo. "El mercado como fuente de innovación en las empresas españolas y la vigilancia

\section{Renovar (o comenzar) la suscripción a "El profesional de la información" es ágil y sen- cillo.}

\section{Usted puede gestionar online su suscripción conectándose a esta página web:}

http://www.elprofesionaldelainformacion. com/suscripciones.html

\section{Si lo desea puede comunicar con nosotros dirigiéndose a esta dirección de correo elec- trónico:} suscripciones@elprofesionaldelainforma cion.com

tecnológica". En: Congreso Rictes, 1999. Santiago de Compostela: Tórculo Artes Gráficas, 1999, pp. 494-501.

Escorsa, Pere. De la vigilancia tecnológica a la inteligencia competitiva. Madrid: Prentice Hall, 2001.

Gestión de la I+D+I: Sistema de vigilancia tecnológica. UNE 166006 EX, mayo 2006.

Giménez, Elea; Román, Adelaida. "Vigilancia tecnológica e inteligencia competitiva: conceptos, profesionales, servicios y fuentes de información". En: El profesional de la información, 2001 , v. 10 , n. 5 , pp. $11-20$.

Guía de vigilancia tecnológica: sistema de información estratégica en las pymes. DZ Centro de Diseño, 2003.

IALE Tecnología. Estudio de vigilancia tecnológica del sector del plástico: informe descriptivo. Ibi: Ayunhtamiento de Ibi, 2003.

Inteligencia económica y tecnológica: guía para principiantes y profesionales. Madrid: Cestisme, 2003.

La vigilancia tecnológica en el sector de los nuevos materiales: biomateriales. Polímeros y plásticos. Cerámicos, vidrios y materiales de construcción. Programa Cyted. IALE Tecnología, 2003.

López, Marta; Mallorquín, Paloma; Vega, Miguel. Genotipado en la salud humana: informe de vigilancia tecnológica. Madrid: Genoma España, 2005

Martín Díaz, Miguel; González Vidal, Francisco (tutor). Diagnóstico tecnológico y diseño y realización de una organización de vigilancia tecnológica para un departamento universitario de enseñanza de ingeniería de telecomunicación. Madrid: M. Martín Díaz, 2005
Millán, Tena; Joaquín y Comai, Alessandro. "El desarrollo de la inteligencia competitiva en España: un recorrido bibliográfico". En: Puzzle, revista hispana de la inteligencia competitiva, 2005 , n. 16 , pp. 4-10.

Morales Luchena, Carlos; Meneses Chaus, Juan Manuel (tutor). Creación de una plataforma de vigilancia tecnológica para comunicaciones móviles $2.5 \mathrm{G}$ y $3 \mathrm{G}$. Madrid: C. Morales, 2004.

Morcillo Ortega, Patricio. Dirección estratégica de la tecnología y la innovación. Madrid: Civitas, 1997.

Oppenheim, Charles; Morris, Anne; McKnight, Cliff; Lowley, S. The evaluation of www search engines. En: Journal of documentation, 2000 , v. 56 , n. 2 , pp. $190-211$.

Palop, Fernando; Vicente, José M. Vigilancia tecnológica e inteligencia competitiva: su potencial para la empresa española. Madrid: Cotec, 1999.

Rouach, Daniel. La veille technologique et l'intelligence économique. Paris: Presses Universitaires de France, 1996.

Tena-Millán, Joaquín; Comai, Alessandro. Inteligencia competitiva y vigilancia tecnológica. Barcelona: Emecom y Puzzle, 2006, 288 pp.

Zaintek. Guía de vigilancia tecnológica: sistema de información estratégica en las pymes.

Zaintek. Manual de implantación de un Sistema de Información Empresarial-SIE.

Lara Rey Vázquez, Ferroatlántica I+D, Departamento de Documentación y Proyectos. lararey@ferroatlantica.es 